\title{
New detection systems at U-120M cyclotron
}

\author{
Jan Novák ${ }^{1, *}$, Martin Ansorge ${ }^{1}$, Pavel Bém ${ }^{1}$, Mitja Majerle ${ }^{1}$, Jaromír Mrázek $^{1}$, Eva Šimečková ${ }^{1}$, and Milan Štefánik $^{1}$ \\ ${ }^{1}$ Nuclear Physics Institute of the Czech Academy of Sciences
}

\begin{abstract}
Intensive neutron beams with energies up to $33 \mathrm{MeV}$ are produced using cyclotron driven broadspectrum and quasi-monoenergetic neutron generators at the NPI CAS. The neutron beams are well characterized with the TOF and PRT measurements. The segmented Fe-CH2 collimator is used to collimate the neutron beam and two detection systems are being developed for the studies of the reactions with neutrons. The first one is the detection system of the charged particles at different angles from the (neutron, charged particle) reactions in a vacuum chamber, the second is an array of four HPGe detectors for the detection of direct and delayed gamma photons produced in reactions of the studied material with neutrons. The advanced bunching system is also being developed at the U120M cyclotron. It will allow to direct a short (few ns long) pulse of accelerated particles to the neutron converter with the $1 \mu$ s delay between bunches. Together with $7 \mathrm{~m}$ long flight path this extension will open new possibilities in the cross section measurements.
\end{abstract}

\section{Introduction}

Isochronous cyclotron U-120M can accelerate light-ion beams $\left(\mathrm{H}^{+}, \mathrm{H}^{-}, \mathrm{D}^{+}, \mathrm{D}^{-},{ }^{3} \mathrm{He}^{+2},{ }^{4} \mathrm{He}^{+2}\right)$. It provides protons in the energy range of $17-35 \mathrm{MeV}$ and currents up to $20 \mu \mathrm{A}$ and deuterons in the energy range of $11-20 \mathrm{MeV}$. In the fast neutron laboratory, two different fast neutron cyclotron-driven sources were developed and one is in the development:

1) Variable-energy broad-spectrum neutron generator. Proton and deuteron beams are suitable to produce a broad-spectrum neutron fields with variable mean energy in the range from 4 to $12 \mathrm{MeV}$ using standard $\mathrm{p} / \mathrm{d}+\mathrm{Be}$ (thick target) source reactions. The integral flux is up to $10^{11} \mathrm{n} / \mathrm{cm}^{2} / \mathrm{s}$.

2) Quasi-monoenergetic neutron generator. The standard ${ }^{7} \mathrm{Li}(\mathrm{p}, \mathrm{n})$ reaction on thin lithium target (with carbon beam stopper) induced by $17-35 \mathrm{MeV}$ proton beam is used for the production of quasi-monoenergetic neutron field (Figure 1). The neutron flux density of the monoenergetic peak is up to $10^{9} \mathrm{n} / \mathrm{cm}^{2} / \mathrm{s}$ and peak energy is up to $33 \mathrm{MeV}$ [1].

3) Variable-energy broad-spectrum neutron generator with high neutron flux density. A thick Be target will be irradiated by $24 \mathrm{MeV}$ protons from the new high current beam cyclotron. The integral flux will be up to $10^{12} \mathrm{n} / \mathrm{cm}^{2} / \mathrm{s}$.

The fast neutron sources are used mainly for neutron cross section measurements, radiation hardness tests, material damage production, neutron activation analysis, detector tests.

Moreover, charged particle cross section measurements are also performed. The equipment is developed for irradiation of samples by protons and deuterons. The

*e-mail: novak@ujf.cas.cz

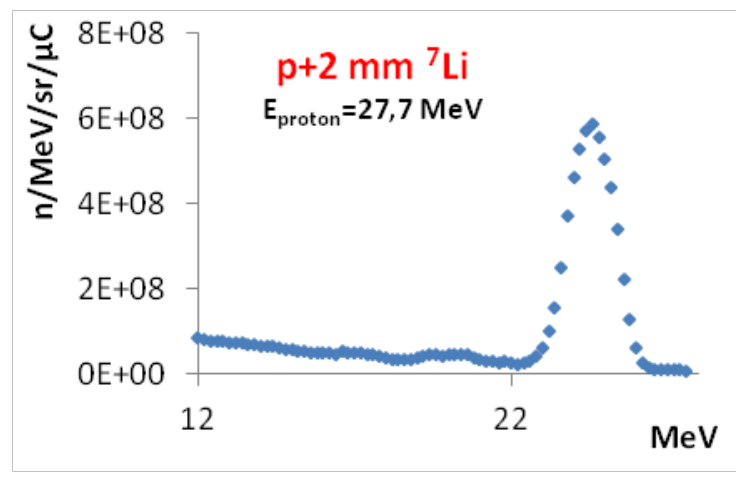

Figure 1. Quasi-monoenergetic neutron spectrum

irradiation chamber is equipped by Faraday cup and by the cooling of samples.

The neutron beam can be shaped using the collimator system and subsequently stopped in the neutron beam dump. The neutron beam spectrum is estimated by the simulation + the measurement of the charged particle beam current. In the case of the Li target station, the measurement of the $\gamma$ activation after irradiation can serve for the spectrum estimation. The last methods for the neutron beam spectrum estimation are TOF and PRT.

\section{Time of flight}

The neutron time of flight measurement in the defined source - detector distance is based on the neutron detection by an organic scintillator (Figure 2).

The scintillator is coupled to the photomultiplier. Its signal is sampled by a $1 \mathrm{GHz}$ digitizer together with the cyclotron radiofrequency for the start time determination. 


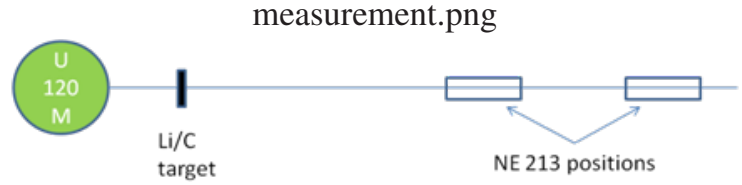

Figure 2. Principle of the TOF measurement

The neutron- $\gamma$ discrimination is performed by the pulseshape analysis. The dynamic threshold method is used for the calculation of the neutron energy spectrum from the measured pulse spectrum. The calculation is based on the counting of pulses with the amplitude higher than the threshold from the $(\mathrm{p}, \mathrm{n})$ scattering to angles between 0 deg. and some fixed angle (Figure 3 ).

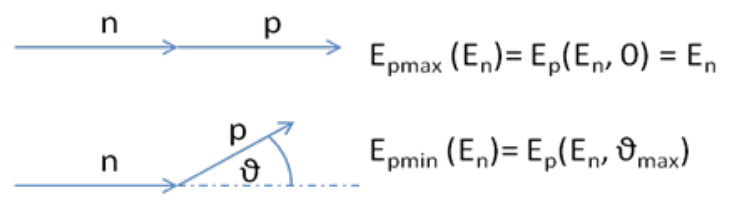

Figure 3. Principle of the dynamic threshold method

The energy dependent scintillator efficiency, for the neutron spectra calculation from the pulse spectrum, Figure 4 , is defined and calculated under the same conditions.

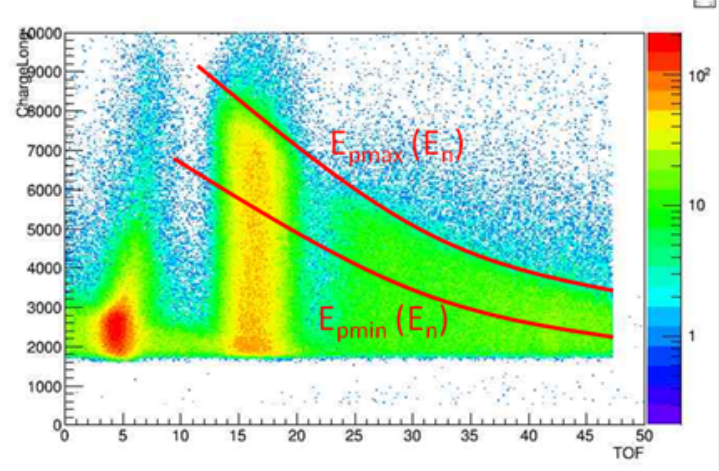

Figure 4. Scintillator pulse response vs. TOF

This approach removes the necessity to know the whole response function of the scintillator, the results are based only on the number of hydrogen atoms in the scintillator and on the cross-section of elastic scattering of neutrons on hydrogen, which are both accurately known. Besides the direct neutron spectra measurements using TOF, the neutron energy can become one of acquisition parameters, in the multichannel coincidence set up. From the two-dimensional measurement of the scintillator response vs. time/energy, the scintillator response function at given time/energy can be extracted (Figure 5).

Currently, a new TOF set up for the significantly longer base and the significantly longer time range is in construction thanks to the bunching system for the U$120 \mathrm{M}$ cyclotron development.

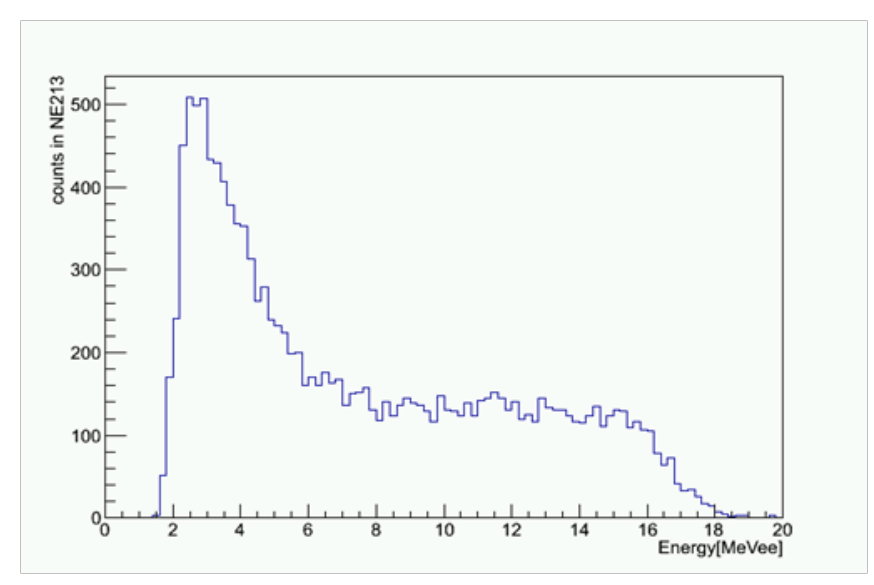

Figure 5. Scintillator response at given time/energy

\section{Proton recoil telescope}

The proton recoil telescope (PRT) was used [2] to determine the neutron spectrum at short distances from the source where TOF method is not applicable. The PRT is based on the detection of protons from the (n,p) scattering in a thin hydrogenated target. By correcting for the energy dependent efficiency, the energy spectrum for the neutrons emitted by the source is determined from the detected proton spectrum, on the kinematics relations basis. Protons are detected at defined angle by the telescope of $\mathrm{Si}$ detectors, Figure 6. Protons are distinguished from other particles by Bethe formula $\mathrm{E} . \Delta \mathrm{E} \sim \mathrm{A} . \mathrm{Z}^{2}$.

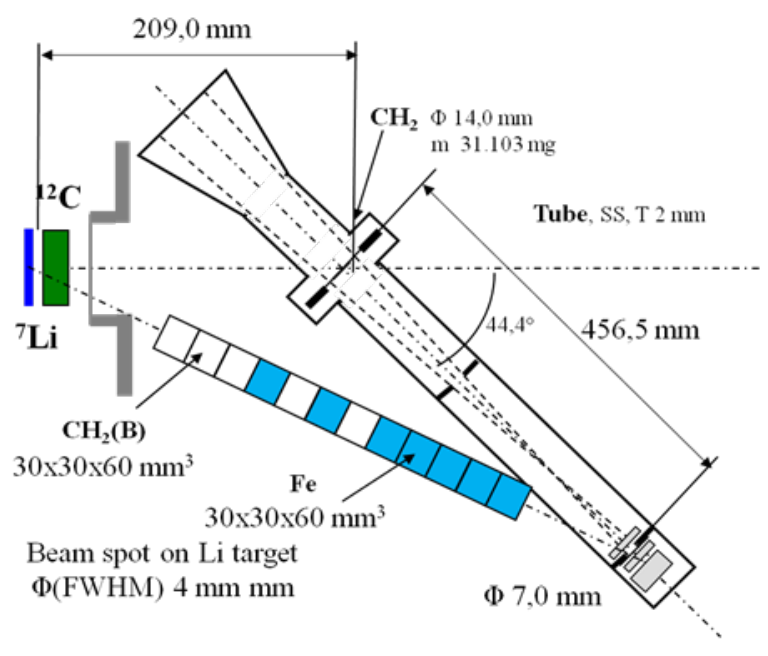

Figure 6. The proton recoil telescope arrangement

The multiplied response functions at different neutron energies are superposed to fit the measured proton-recoil spectrum. Multiplication factors pertaining to response functions create the resulting neutron spectrum. The response functions were calculated analytically using the full geometry arrangement of the target-radiator-detector setup. The MCNPX simulated neutron spectrum at the radiator position, the neutron spectrum measured by TOF data by Uwamino [3] extrapolated to the radiator position 
and the neutron spectrum deconvoluted from the experimental net-effect proton spectrum (full squares) [2] are compared in Figure 7.

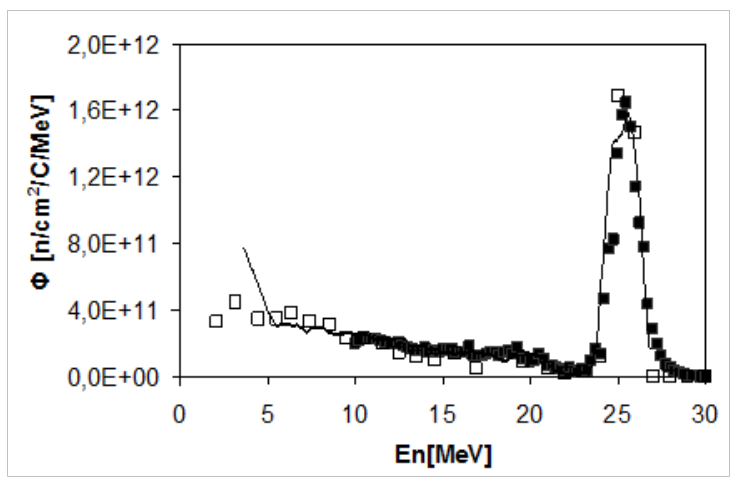

Figure 7. Simulated neutron spectrum (empty squares), TOF data (line) and simplified deconvolution of proton-recoil spectrum (full squares)

The measured spectra from PRT-data deconvolution agree with MCNPX simulation [4] within 10\%. Protonrecoil-telescope method was found suitable for validation of MCNPX-simulated neutron flux at foil position. Moreover, the MCNPX simulation reproduces well the measured data from proton-recoil-telescope method.

\section{Detection systems at U-120M cyclotron}

For the reaction product measurement, one detection system is used and two systems are in construction.

\subsection{Measurement of $\gamma$ activation after irradiation}

The targets from target stations, as well as samples irradiated by fast neutrons, are transported by pneumatic transport system to the HPGe detector station in tens of seconds after irradiation (Figure 8). The residual products are also measureable after irradiation with charged particles.

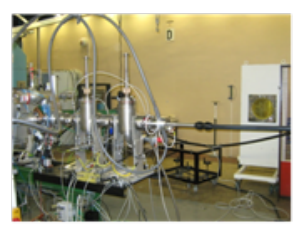

Pneumatic post system is used for fast transport of the irradiated samples to the irradiated samples to the detectors.

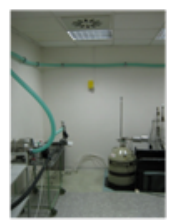

Figure 8. Measurement of $\gamma$ activation after irradiation

\subsection{System for the direct detection of charged particles, which are produced in reaction with neutrons}

The system is in construction. It consists of a large vacuum chamber with a target in the center (Figure 9). The electronically controlled manipulator allows to change up to 4 targets without opening the chamber.

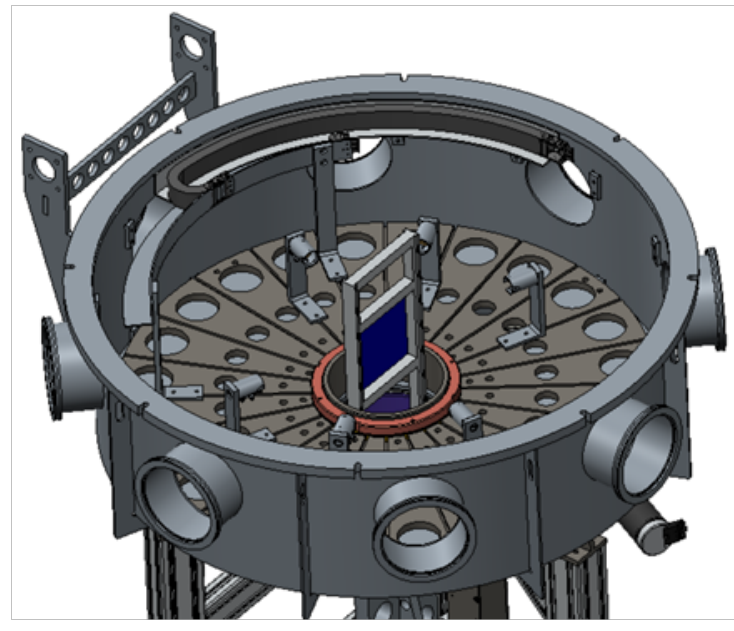

Figure 9. Large vacuum chamber

The dE-E telescopes for the charged particle detection are composed of thin and thick silicon detectors to distinguish the type of the charged particle based on Bethe's formula. The telescopes are placed on a rotating table at different angles, outside the reach of the neutron beam. The rotation is remotely controlled by electronics. The above-mentioned bunching system for the U-120 M cyclotron as well as the new TOF set up will make the detection system usable for direct cross-section measurements of (neutron, charged particle) reactions. By measuring angular distributions of the charged particles, a more detailed information on the reaction mechanism will be obtained. This will extend the laboratory know-how from measurements to nuclear reaction modeling.

\subsection{System for the direct detection of $\gamma$ photons, produced in reaction with neutrons}

The system is in construction. Four positionable HPGe detectors (Figure 10) are placed around the irradiated sample at different angles in respect to the beam direction.

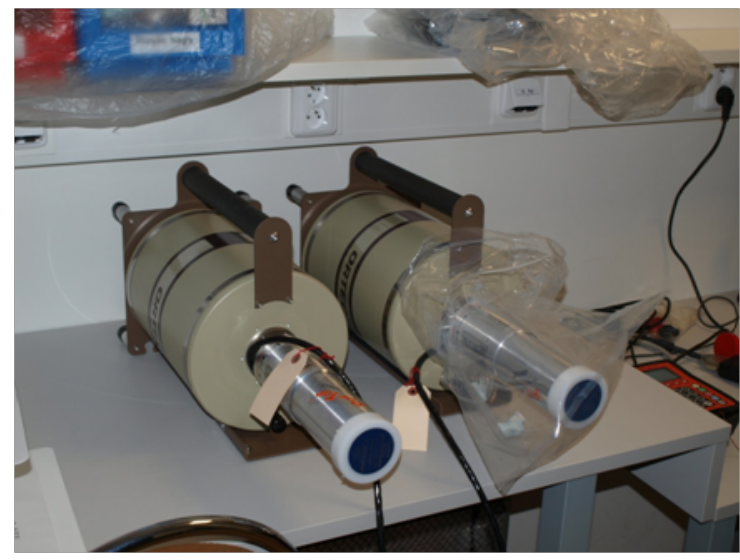

Figure 10. Positionable HPGe detectors

The samples are irradiated by fast neutron beam, whereas the $\gamma$ detector position is chosen to be outside the 
reach of the direct neutrons. The system is usable also for the measurement with charged particles. So far, the $\gamma$ measurement can start some tens of seconds after irradiation. The new system will allow to measure the residuals with decay times down to ms. Moreover, the bunching system will make the online gamma spectrometer usable for the direct cross-section measurement of the (neutron, $\mathrm{x} \gamma$ ) reactions. In combination with the neutron generators, the detection system will be most efficient in the neutron energy range of 10-33 MeV.

\section{Conclusions}

The variable-energy broad-spectrum neutron generator and the quasi-monoenergetic neutron generator are successfully used for neutron experiments. The accuracy of the produced fast neutron spectra determination is continually increasing by the use of improved measurement techniques. The experience of using the current generators is used during construction of a new broad-spectrum target station. The neutron TOF method has been successfully used. The largely improved TOF set up system is in construction. The system of the off-beam $\gamma$ detection after neutron or charged particle irradiation is currently commonly used. Moreover, two new detection systems are in construction. First of them is the system for the direct detection of charged particles, which are produced in reaction with neutrons. The second one is the system for the direct detection of $\gamma$ photons, which are produced in reaction with neutrons.

\section{References}

[1] P. Bém, V. Burjan, M. Götz, M. Honusek, U. Fischer, V. Kroha, J. Novák, S.P. Simakov and E. Šimečková, Int. Conf. on Nucl. Data for Sci. \& Techn., Nice (2007)

[2] J. Novák et al., Journal of the Korean Physical Society 59, 1157-1580 (2011)

[3] Y. Uwamino et al., Nucl. Instr. Meth., A389, 463 (1997)

[4] S.P. Simakov et al., Journal of the Korean Physical Society, 59, 1856-1859 (2011) 